\title{
Routine deep vein thrombosis screening after lung transplantation: Incidence and risk factors
}

\author{
Ahmed Jorge, PhD, ${ }^{\mathrm{a}}$ Pablo G. Sanchez, MD, PhD, ${ }^{\mathrm{a}}$ J. W. Awori Hayanga, MD, MPH, \\ Joseph M. Pilewski, MD, ${ }^{\mathrm{c}}$ Mathew Morrell, MD ${ }^{\mathrm{c}}$ Marie Tuft, BS, ${ }^{\mathrm{d}} \mathrm{John}$ Ryan, PhD, ${ }^{\mathrm{a}}$ and \\ Jonathan D'Cunha, $\mathrm{MD}, \mathrm{PhD}^{\mathrm{e}}$
}

\begin{abstract}
Background: Deep vein thrombosis (DVT) remains a common complication following lung transplantation despite universal routine DVT screening. Moreover, many of the previously reported risk factors are incompletely defined. We sought to explore the influence of DVT screening and to more definitively assess predisposing risk factors.
\end{abstract}

Methods: A single-institution, retrospective, cohort study of 1141 patients undergoing lung transplantation from January 1, 2005, to December 31, 2014, was performed evaluating for the rate of DVT. Patients were given prophylactic subcutaneous heparin postoperatively. DVT events were noted if they occurred before 90 days after transplant. We compared DVT rates before and after 2008 when universal screening was implemented. We also evaluated the timing of DVT event and location (above the knee vs below the knee). DVTs were treated with standard anticoagulation therapy or an inferior cava filter when patients were unable to tolerate anticoagulation treatment. Univariable and multivariable models were used to identify risk factors for occurrence. A propensity match was performed to match groups across the eras, and a Cox regression was performed to identify differences in 1-year survival trajectory between cohorts.

Results: The rates of DVT before and after routine screening were $8.8 \%$ (36 DVT out of 412 transplants) and $17.3 \%$ (126 out of 729 transplants), respectively. These 2 rates were significantly different $(P<.01)$; moreover, the observed DVT incidence per year was not significantly different across the 6 years after universal DVT screening was implemented ( $P>.90$ for all comparisons). Observed DVT incidence at day 0 and day 14 were $3.8 \%$ and $3.8 \%$, respectively, for the cohort before DVT protocols were established. Observed DVT incidence for the cohort after protocols were established at the same time points was $8.7 \%$ and $3.7 \%$, respectively. Univariable analysis revealed that significant factors associated with a DVT include hypercholesterolemia (odds ratio [OR], 6.90; $95 \%$ confidence interval $[\mathrm{CI}], 1.82-26.13 ; P<.01)$, the number of days in the intensive care unit $(\mathrm{OR}, 1.03 ; 95 \% \mathrm{CI}, 1.00-1.01 ; P<.01)$, and the length of stay in the hospital (OR, 1.01; 95\% CI, 1.01-1.02; $P<.01)$, whereas having quit smoking (vs never smoked) was associated with a decrease in DVT development (OR, 0.50; 95\% CI, 0.33-0.75; $P<.01$ ). Multivariable analysis revealed 2 significant variables: hypercholesterolemia (OR, 8.13; 95\% CI, 1.22-54.37; $P=.03)$ and length of stay (OR, $1.03 ; 95 \% \mathrm{CI}, 1.01-1.05 ; P<.01)$. There was a trend for better 1-year survival in the post-2008 era $(\operatorname{Exp}[\beta], 1.49 ; P=.09)$.

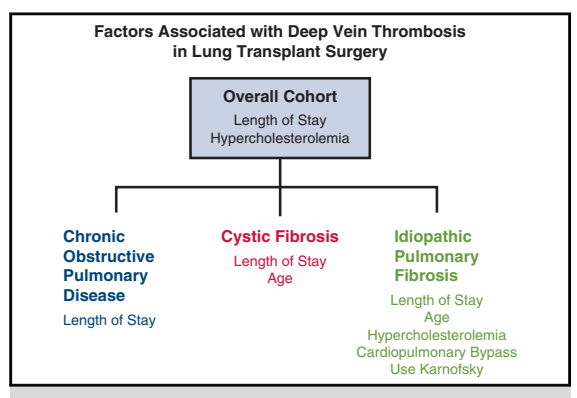

Ultrasound image of a DVT.

\section{Central Message}

Venous thromboembolism is a common complication following lung transplantation. This analysis showed that postoperative screening identified increased rates of detection.

\section{Perspective}

Venous thromboembolism is a common complication following lung transplantation. This retrospective database analysis demonstrated significantly increased detection rates following implementation of postoperative universal screening. It is our perspective that programs performing lung transplantation consider instituting screening programs to aid in reducing the morbidity of this complication.

See Commentaries on pages 1151, 1152 , and 1154.
From the ${ }^{\mathrm{a} D i v i s i o n}$ of Lung Transplantation and Lung Failure, Department of Cardiothoracic Surgery, University of Pittsburgh Medical Center; ' $D$ Division of Pulmonary, Allergy, and Critical Care Medicine, Department of Medicine, School of Medicine; and ${ }^{\mathrm{d}}$ Department of Biostatistics, Graduate School of Public Health, University of Pittsburgh, Pittsburgh, Pa; ${ }^{b}$ Department of Cardiovascular and Thoracic Surgery, West Virginia University, Morgantown, WVa; and ${ }^{\mathrm{e}}$ Department of Cardiothoracic Surgery, Mayo Clinic Arizona, Phoenix, Ariz.

Received for publication Nov 21, 2018; revisions received July 31, 2019; accepted for publication Aug 4, 2019; available ahead of print Dec 12, 2019.
Address for reprints: Jonathan D'Cunha, MD, PhD, Department of Cardiothoracic Surgery, Mayo Clinic Arizona, 5777 E Mayo Blvd, Phoenix, AZ 85054 (E-mail: DCunha.Jonathan@mayo.edu). 0022-5223/\$36.00

Copyright (C) 2019 Published by Elsevier Inc. on behalf of The American Association for Thoracic Surgery

https://doi.org/10.1016/j.jtcvs.2019.08.030 

Abbreviations and Acronyms
COPD $=$ chronic obstructive pulmonary disorder
$\mathrm{DM}=$ diabetes mellitus
DVT $=$ deep vein thrombosis
$\mathrm{ECMO}=$ extracorporeal membrane oxygenation
IVC = inferior vena cava
KPS $=$ Karnofsky Performance Scale
LAS = lung allocation score
LOS = length of stay
LTx = lung transplantation

Conclusions: The rate of DVT diagnosis significantly increased after universal DVT screening was implemented. Furthermore, those patients undergoing lung transplantation with extended length of stay and hypercholesterolemia were prone to increased rates of DVT. There was a trend toward better 1-year survival in DVT-screened patients, suggesting DVT screening may result in beneficial outcomes. (J Thorac Cardiovasc Surg 2020;159:1142-50)

Lung transplantation (LTx) is the only viable option as a lifesaving procedure for those with end-stage lung disease. Keeping patients from developing significant complications postoperatively is an important component for short-term and long-term success of the operation. A deep vein thrombosis (DVT) is an important postoperative complication in this population as it is potentially a source of significant morbidity. Although the estimated DVT incidence in cardiac surgery and heart transplantation is $5 \%$ and $14.8 \%$, respectively, ${ }^{1-3}$ the estimated incidence in LTx has been variably reported from $8 \%$ to $27 \% .{ }^{4}$ Reported risk factors for the development of DVT after LTx include advanced age, obesity, diabetes mellitus (DM), cardiopulmonary bypass, pneumonia, prothrombotic state, and mammalian target of rapamycin immunosuppression therapy (eg, sirolimus and everolimus). ${ }^{4-6}$

The studies published to date typically report smaller sample sizes with significant variability in reported findings and heterogeneity in terms of the approach to screening patients who undergo LTx..$^{4-6}$ We sought to investigate, with a significant larger population sample size, significant risk factors for the development of DVT and whether implementation of a DVT routine screening protocol would result in a significant increase in DVTs identified. We also sought to estimate the treatment effect of DVT screening on 1-year survival using propensity analysis.

\section{METHODS}

We conducted a single-center, retrospective, cohort study of all LTx recipients who underwent LTx from January 1, 2005, to December 31,
2014. The study was approved by the University of Pittsburgh Institutional Review Board. Follow-up data on all patients were obtained until July 2, 2015. Demographic characteristic indicators, procedure variables, and postoperative factors were systematically collected from medical records.

At our institution, lower-extremity DVT screening protocols changed in 2008 from no screening during the pre-2008 era to screening every patient undergoing LTx on day 0 and day 14 after LTx. We selected these time points empirically based on the fact that our team was interested in early identification of any DVT and identification of a DVT before discharge. The early time point was an effort to identify a DVT in a population that may be at high risk due to premorbid medical conditions and sedentary lifestyle. The day-14 time point was selected in an effort to identify a problem before a patient leaves the hospital because we noticed significant variability in the mobility patterns of our postoperative patients. There was no clinical evidence that guided the selection of this screening time point. Karnofsky Performance Scale (KPS) scores were recorded and binarized as above or below $80-\mathrm{a}$ score that signifies that a patient is able to carry on normal activity and work and needs no special care. ${ }^{7}$ Patients were not scanned a second time if discharged before day $14(n=43 ; 6 \%$ of the entire cohort after protocols were established). Screening consisted of a duplex ultrasound performed by a certified technician from the ultrasound division. A DVT episode was noted only if it occurred within a 90-day window after surgery. Thirty-two patients were excluded because they developed a DVT after 90 days. Those patients who developed a DVT within the 90-day post-LTx period while taking sirolimus or everolimus were excluded $(n=28)$ because these mammalian target of rapamycin immunosuppression therapy inhibitors are known to predispose patients to DVT. ${ }^{2}$

Screening for upper extremity DVTs did not take place, and upper extremities were only evaluated when symptoms developed. All patients were subjected to bilateral lower extremity compression devices after surgery and, if no contraindications existed, they were prescribed $5000 \mathrm{U}$ heparin thrice daily throughout their entire hospitalization. In the case that a DVT was identified, affected patients were treated with anticoagulation therapy according to standard clinical pathways that involved intravenous heparin and transition to oral warfarin with a goal international normalize ratio of 2 to 3 . This warfarin regimen continued for 6 months. Patients who could not tolerate anticoagulation therapy had an inferior vena cava (IVC) filter placed. After discharge, prophylactic anticoagulation therapy was not routinely prescribed. We excluded DVTs that developed after 90 days to confine our analysis to those related only to the perioperative period. Patients receiving extracorporeal membrane oxygenation (ECMO) postoperatively received the same screening protocol. No antithrombotic agent was given preoperatively.

The univariable analyses were performed using Pearson $\chi^{2}$ tests for categorical variables, $t$ test (2-sided) for continuous normal variables, and a Wilcoxon rank-sum test for nonnormal continuous variables. The resulting variables that satisfied normality conditions and variables that were skewed in their distribution were noted appropriately. Variables with $P$ value $<.2$ from the univariable analyses were entered into a multivariable logistic regression model in 1 step (forced entry) with presence (or absence) of DVT within 90 days as the outcome. To build a more parsimonious model, backward selection was performed on the full model and variables with $P>.05$ were excluded from the model. In addition to the multivariable model, a Kaplan-Meier analysis was performed and stratified for patients who developed a DVT within 90 days and censoring deaths before 90 days. A log-rank test was used to check for differences in survival trajectory between the subgroups. Mean DVT event rate was calculated by observed DVT incidence per year. Analysis of variance was used to determine whether or not there were differences in DVT rates across years. Finally, to estimate the effect of screening on DVT detection, a propensity score match was conducted. Propensity scores were determined based on factors that were significantly associated with 
DVT in a multivariable regression. Matching was performed using nearest neighbor matching with matches in random order. Cases with propensity scores $<0.10$ or $>0.90$ were removed from the dataset $(\mathrm{n}=8)$. Treatment effect was calculated by multivariable logistic regression, including the treatment indicator and propensity score as a covariate with 1-year survival as the outcome. We also conducted a Cox regression with propensity scores as a covariate to determine the treatment effect on 1-year survival.

\section{RESULTS}

The overall patient population consisted of 1141 patients from 2005 to 2014 . We then divided this population into 2 groups: before universal DVT protocols were established $(\mathrm{n}=412)$ and after $(\mathrm{n}=729)$. We analyzed these groups, as shown in Table 1, to identify differences with respect to demographic, procedure-related, and postoperative variables. No significant differences were found with the exception of preoperative creatinine level, which was higher in the screened group.

The mean overall observed DVT incidence (including both upper and lower DVTs) and the mean observed DVT incidence for lower DVTs only, were significantly different before and after protocols were established (Figure 1). In contrast, the mean observed DVT incidence for upper DVTs was not significantly different before and after protocols were established (Figure 1). Observed DVT incidence at day 0 and day 14 was $3.8 \%$ and $3.8 \%$, respectively (all symptomatic), for the cohort before DVT protocols were established, whereas the observed DVT incidence for the cohort after protocols were established at the same time points were $8.7 \%$ and $3.7 \%$, respectively (majority asymptomatic). For the cohort after protocols were established, rate of DVT occurrence was $2.6 \%$ before day 14 (not including day 0) and $1.4 \%$ after day 14 up until time of discharge. There were no asymptomatic DVTs on day 14 that were treated and there were no patients who developed a pulmonary embolism without a DVT on day 14 . Three patients were treated with an IVC filter and none had a preexisting IVC filter.

To identify potential confounders, multivariable logistic regression was performed with DVT as the outcome.

TABLE 1. Patient demographic characteristics before and after the implementation of deep vein thrombosis protocols

\begin{tabular}{|c|c|c|c|}
\hline Characteristic & $\begin{array}{l}\text { All transplants before protocols } \\
\qquad(\mathrm{n}=\mathbf{4 1 2})\end{array}$ & $\begin{array}{l}\text { All transplants after protocols } \\
\qquad(\mathbf{n}=\mathbf{7 2 9})\end{array}$ & $P$ value \\
\hline Age (y) & $54.5 \pm 13.9$ & $56.1 \pm 13.8$ & .07 \\
\hline BMI & $25.9 \pm 5.5$ & $25.7 \pm 5.1$ & .56 \\
\hline Male sex vs female sex & 54.4 & 58.3 & .20 \\
\hline White race vs black race & 94.5 & 93.2 & .40 \\
\hline Quit smoking vs never smoked & 68.2 & 68.6 & .90 \\
\hline Diabetes & 20.5 & 17.8 & .27 \\
\hline Hypertension & 21.5 & 26.4 & .07 \\
\hline Hypercholesterolemia & 0.25 & 1.34 & .11 \\
\hline Preoperative creatinine (mg/dL) & $0.91 \pm 0.30$ & $0.86 \pm 0.30$ & $<.01$ \\
\hline Postoperative creatinine (mg/dL) & $2.14 \pm 1.70$ & $2.14 \pm 1.60$ & .97 \\
\hline CPB use & 34.2 & 32.2 & .49 \\
\hline CPB time (min) & $232 \pm 87$ & $230 \pm 91$ & .81 \\
\hline ECMO use & N/A & 14.3 & N/A \\
\hline ECMO time (min) & N/A & $309.9 \pm 144$ & N/A \\
\hline ICU stay (d) & $14.8 \pm 24$ & $15.1 \pm 24$ & .86 \\
\hline CMV immunoglobulin G threshold* & 54.2 & 55.7 & .98 \\
\hline Hospital LOS (d) & $35.8 \pm 32.5$ & $36.3 \pm 33.3$ & .82 \\
\hline Karnofsky Performance Scale score $\geq 80$ vs $\leq 70$ & N/A & 69.4 & N/A \\
\hline Bronchiectasis & 0.9 & 1.1 & N/A \\
\hline COPD/emphysema & 36 & 33 & .35 \\
\hline Cystic fibrosis & 14 & 12 & .51 \\
\hline Idiopathic pulmonary fibrosis & 30 & 34 & .17 \\
\hline Sarcoidosis & 2.9 & 2.3 & .59 \\
\hline Scleroderma & 3.1 & 3.9 & .44 \\
\hline
\end{tabular}

Values are presented as $\%$ of the total for that column or mean \pm standard deviation. $B M I$, Body mass index, $C P B$, cardiopulmonary bypass; $E C M O$, extracorporeal mechanical oxygenation; $N / A$, not available; $I C U$, intensive care unit; $C M V$, cytomegalovirus; $L O S$, length of stay; $C O P D$, chronic obstructive pulmonary disease. *Positive $>4$. 


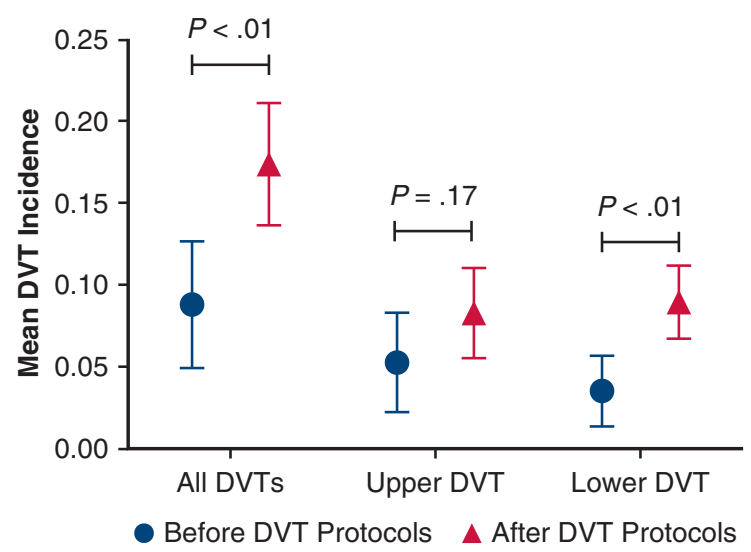

FIGURE 1. Overall deep vein thrombosis (DVT) mean DVT incidence, upper body DVT incidence, and lower body DVT incidence for all diagnoses before (blue circles) and after (red triangles) universal DVT screening protocols were established. There were significant differences in the overall and lower body incidences when comparing before and after DVT protocols were established $(P=.005$ and $P=.003$, respectively).

Propensity score matching was conducted utilizing age, partial thromboplastin time, DM before transplant, tobacco use history, and hypercholesterolemia. After propensity matching, individuals who were screened were more than twice as likely to have a DVT detected than those who were not screened $(\beta, 0.78 \pm 0.30 ; \operatorname{Exp}[\beta], 2.18$; $P=.010)$. The Cox regression with propensity scores revealed a trend toward higher 1-year survival in the screened cohort $(\beta, 0.40 \pm 0.24 ; \operatorname{Exp}[\beta], 1.49 ; P=.09)$.

From this point forward, we exclusively analyzed the cohort after DVT protocols were established because this is the group with universal DVT screening protocols and thus more relevant to our discussion. We subdivided the screened cohort into 2 subgroups: if a DVT developed within the 90-day window $(n=127)$ or not $(n=602)$. The group after DVT protocols were established had a median age of 56 years, ranging from 14 to 81 years. The majority of the cohort was composed of white $(93 \%)$ and male $(58 \%)$ patients. At the time of transplantation, 587 patients had a median lung allocation score (LAS) of 42 , with lower and upper quartiles at 35 to 63 , respectively. Notably, patients with a higher LAS and a higher length of stay (LOS) were associated with higher rates of DVT at or before 90 days than patients with a lower LAS (odds ratio [OR], $1.01 ; 95 \%$ confidence interval $[\mathrm{CI}], 1.00-1.02 ; P<.06$ ); however, this association was not significant. DVT rates were not correlated to year $(r=0.59 ; P=.73)$

Pre-existing conditions such as hypertension, DM, and hypercholesterolemia comprised $26 \%, 18 \%$, and $1 \%$, respectively, of the total patient population. Three diagnoses, namely, chronic obstructive pulmonary disorder (COPD), cystic fibrosis, and idiopathic pulmonary fibrosis accounted for $79 \%$ of cases. A vast majority of cases

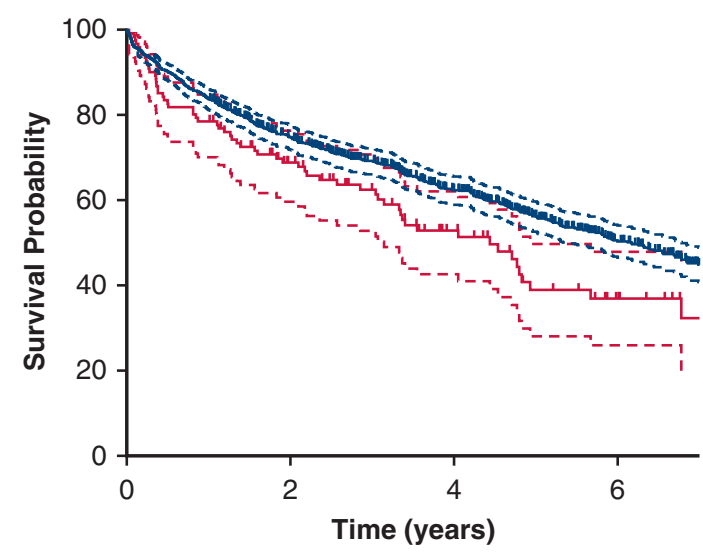

$\begin{array}{ccccc}\text { At risk } & & & \\ \text { - no DVT } & 602 & 415 & 272 & 146 \\ \text { - DVT } & 127 & 70 & 36 & 14\end{array}$

FIGURE 2. Percent survival (solid line) and confidence interval (dashed line) as a function of time (years) for the without (blue) and with (red) deep vein thrombosis $(D V T)$ episode groups after lung transplant surgery estimated with the Kaplan-Meier method revealed that both curves are not significantly different $(P=.44)$. Included table highlights each group of patients at risk at 2-year time intervals.

$(95 \%)$ were patients receiving their first graft (vs patients receiving their second or third graft), whereas the majority of transplants $(80 \%)$ were bilateral lung operations. Cardiopulmonary bypass and ECMO were used in $32 \%$ and $14 \%$ of cases, respectively.

DVT at or before 90 days was found in 127 (17\%) out of 729 LTx recipients, $72(57 \%)$ were found in the lower extremity (55 above and 17 below the knee), and 55 (43\%) were found in an upper extremity. A total of 14 DVTs $(11 \%)$ were associated with a peripherally inserted central catheter and all of these were found in an upper extremity. The median time to develop DVT was 20 days with an interquartile range (IQR) 6 to 26 days. There was an increase in observed DVT incidence around day 0 and day 14 when compared with before protocols were implemented; however, DVTs were still discovered after day 14 and up to 90 days and thus it is reflected on the median time to DVT. A survival probability analysis estimated using the Kaplan-Meier method showed no association between DVT before or after 90 days and 1-year survival $(P=.44)$. Performing the same analysis, all comparisons within subgroups (eg, in the COPD subgroups: DVT vs non-DVT) revealed no significant differences in survival rates. Extending the survival analysis to the total study duration revealed that there was a significant difference in survival between the DVT and non-DVT groups (non-DVT group median survival $=6.2$ years vs DVT group survival $=4.4$ years $)(P=.02)$ (Figure 2$)$. Graft failure accounted for $3.9 \%$ of patients in the non-DVT group, 
whereas it accounted for $4.7 \%$ in the DVT group (not significantly different at $P=.37$ ).

Results from our univariable analysis of patients with any diagnosis are summarized along with their $P$ value (Table 2). Factors such as age, body mass index, sex, race, $\mathrm{DM}$, or hypertension were not significantly associated between the DVT and non-DVT subgroups. Paradoxically, patients who had quit smoking at some point before transplant were significantly less likely to develop DVT (71\%) when compared with patients who had never smoked $(55 \%)$ $(P<.01)$. A history of hypercholesterolemia was associated with an increased incidence of DVT $(P<.04)$. Moreover, variables such as the number of days in the intensive care unit (14 vs 19 days; $P=.038$ ) and LOS (34 vs 50 days; $P<.01)$ were significantly associated with the development of DVT. A KPS score $\geq 80$ was associated with decreased incidence of DVT $(P=.02)$.

Patients with a diagnosis of COPD, cystic fibrosis, or idiopathic pulmonary fibrosis were separated and analyzed in a similar fashion as with the overall group (Table 3).
Longer length of stay was a significant predictor of DVT for all 3 diagnoses $(P=.03, P=.03$, and $P=.02$, respectively). Within patients with a cystic fibrosis or idiopathic pulmonary fibrosis diagnosis, older age was associated with the development of a DVT. Moreover, patients with a COPD diagnosis were less associated with the development of a DVT $(P<.01)$. Finally, the presence of hypercholesterolemia, cardiopulmonary bypass use, and a KPS score $\leq 70$ were associated with the development of a DVT.

Multivariable analysis found that pretransplant hypercholesterolemia (OR, 8.13; 95\% CI, 1.22-54.37; $P=.03$ ) and LOS (OR, 1.02; $P<.01)$ were factors associated with DVT (Table 4). ECMO was included as a variable but did not reach significance in the multivariable analysis $(P=.13)$. These results have been graphically summarized (Figure 3), where the overall cohort risk for DVT is associated with LOS and hypercholesterolemia. Furthermore, LOS remains a significant risk factor when the cohort is separated into the 3 most common diagnoses, whereas other risk factors are differentially represented (see Figure 3).

TABLE 2. Patient demographic characteristics and univariable analysis for all diagnoses

\begin{tabular}{|c|c|c|c|c|}
\hline Characteristic & $\begin{array}{l}\text { All transplants } \\
\qquad(N=729)\end{array}$ & $\begin{array}{l}\text { Did not develop DVT } \\
\quad(n=602)\end{array}$ & $\begin{array}{l}\text { Developed DVT } \\
\quad(\mathbf{n}=127)\end{array}$ & $P$ value \\
\hline Age $(y)$ & $56.1 \pm 13.8$ & $55.9 \pm 13.8$ & $57.28 \pm 14.1$ & .31 \\
\hline BMI & $25.7 \pm 5.1$ & $25.6 \pm 5.0$ & $26.20 \pm 5.6$ & .25 \\
\hline Male sex & 58.3 & 57.5 & 62.2 & .35 \\
\hline White race & 93.2 & 93.1 & 93.9 & .74 \\
\hline Quit smoking vs never smoked & 68.6 & 71.1 & 55.0 & $<.01$ \\
\hline Diabetes & 17.8 & 17.11 & 21.5 & .28 \\
\hline Hypertension & 26.4 & 26.4 & 26.1 & .95 \\
\hline Hypercholesterolemia & 1.34 & 0.71 & 4.67 & $<.01$ \\
\hline Preoperative creatinine $(\mathrm{mg} / \mathrm{dL})$ & $0.86 \pm 0.3$ & $0.85 \pm 0.3$ & $0.90 \pm 0.3$ & .15 \\
\hline Postoperative creatinine (mg/dL) & $2.14 \pm 1.6$ & $2.10 \pm 1.4$ & $2.35 \pm 2.4$ & .15 \\
\hline $\mathrm{CPB}$ use & 32.2 & 32.7 & 29.4 & .47 \\
\hline CPB time (min) & $230.32 \pm 90.6$ & $227.50 \pm 87.3$ & $246.46 \pm 107.5$ & .25 \\
\hline ECMO use & 14.2 & 13.4 & 18.5 & .15 \\
\hline ECMO time (min) & $309.9 \pm 144.0$ & $313.7 \pm 133.7$ & $295.8 \pm 180.0$ & .60 \\
\hline ICU stay (d) & $15.1 \pm 24.2$ & $14.2 \pm 22.8$ & $19.4 \pm 30.0$ & .04 \\
\hline CMV immunoglobulin G threshold* & 55.7 & 55.1 & 59.4 & .42 \\
\hline Hospital LOS (d) & $36.3 \pm 33.3$ & $33.6 \pm 29.6$ & $49.8 \pm 45.7$ & $<.01$ \\
\hline Karnofsky Performance Scale score $\geq 80$ vs $\leq 70$ & 69.4 & 71.3 & 59.1 & .02 \\
\hline Bronchiectasis & 1.1 & 0.8 & 2.5 & .12 \\
\hline COPD/emphysema & 33 & 35 & 20 & $<.01$ \\
\hline Cystic fibrosis & 12 & 11 & 18 & .05 \\
\hline Idiopathic pulmonary fibrosis & 34 & 33 & 40 & .12 \\
\hline Sarcoidosis & 2.3 & 2.6 & 0.8 & .26 \\
\hline Scleroderma & 3.9 & 4.1 & 3.4 & .71 \\
\hline
\end{tabular}

Values are given as \% of the total for that column or mean \pm standard deviation. $D V T$, Deep vein thrombosis; $B M I$, body mass index; $C P B$, cardiopulmonary bypass; $E C M O$, extracorporeal mechanical oxygenation; $I C U$, intensive care unit; $C M V$, cytomegalovirus, $L O S$, length of stay; $C O P D$, chronic obstructive pulmonary disease. *Positive $>4$. 
TABLE 3. Patient demographic characteristics and univariable analysis for chronic obstructive pulmonary disease, cystic fibrosis, and idiopathic pulmonary fibrosis diagnoses, respectively. Only significant variables are shown, although all variables investigated in Table 2 were tested

\begin{tabular}{|c|c|c|c|c|}
\hline Characteristic & All transplants & Did not develop DVT & Developed DVT & $P$ value \\
\hline Chronic obstructive pulmonary disease & $\mathrm{n}=240$ & $\mathrm{n}=214$ & $\mathrm{n}=26$ & \\
\hline Hospital length of stay (d) & $34.24 \pm 31.4$ & $33.41 \pm 20.2$ & $34.41 \pm 33.3$ & .03 \\
\hline Cystic fibrosis & $\mathrm{n}=89$ & $\mathrm{n}=67$ & $\mathrm{n}=22$ & \\
\hline Age $(y)$ & $31.45 \pm 9.1$ & $31.36 \pm 9.5$ & $32.00 \pm 5.8$ & .03 \\
\hline Hospital length of stay (d) & $32.29 \pm 18.7$ & $29.82 \pm 10.3$ & $32.66 \pm 19.7$ & .01 \\
\hline Idiopathic pulmonary fibrosis & $\mathrm{n}=249$ & $\mathrm{n}=198$ & $\mathrm{n}=51$ & \\
\hline Age (y) & $62.24 \pm 9.3$ & $60.41 \pm 10.9$ & $62.63 \pm 8.8$ & .02 \\
\hline Hypercholesterolemia & 2.17 & 2.13 & 2.38 & .01 \\
\hline Cardiopulmonary bypass use & 33.33 & 33.17 & 34.09 & .04 \\
\hline Hospital length of stay (d) & $38.81 \pm 42.6$ & $32.24 \pm 23.8$ & $40.18 \pm 45.5$ & .02 \\
\hline Karnofsky Performance Scale score $\geq 80$ vs $\leq 70$ & 67.16 & 72.97 & 65.85 & .07 \\
\hline
\end{tabular}

Values are presented as \% of the total for that column or mean \pm standard deviation. DVT, Deep vein thrombosis.

The DVT rate per year was not significantly different. Finally, of 127 recipients who developed a single DVT, 15 $(12 \%)$ developed a second DVT (Table 5). For these patients, the median time to develop their first DVT was 12 days (IQR, 3-24 days), the median time to develop their second DVT was 124 days (IQR, 20-233 days), and their median time to death was 16 months (IQR, 13-27 months). The majority of these patients were diagnosed with idiopathic pulmonary fibrosis $(53 \%)$ and $43 \%$ were former cigarette smokers. None of these patients had pretransplant hypercholesterolemia.

\section{DISCUSSION}

Patients with end-stage lung disease undergoing LTx face a multitude of infectious and noninfectious complications. Venous thromboembolism is among those common complications. Whereas pulmonary embolism and DVT are the most common manifestations of a venous thromboembolism, we sought to focus primarily on the risk factors associated with the development of DVT in the LTx population and to determine whether universal DVT screening protocols are advantageous. We found that our DVT discovery rate increased significantly from $9 \%$ to $17 \%$ after universal DVT protocols were implemented. After propensity matching across the 2 eras, there was a trend toward improved 1 -year survival in the screened cohort, suggesting a possible survival benefit to the increased DVT detection.

We hypothesize that the increase in DVT detection is not only due to an increase in DVT diagnoses on day 0 and day

TABLE 4. Multivariable regression model to first deep vein thrombosis episode for any diagnosis

\begin{tabular}{lcc}
\hline \multicolumn{1}{c}{ Variable } & $\begin{array}{c}\text { Odds ratio }(\mathbf{9 5} \% \text { confidence } \\
\text { interval) }\end{array}$ & $\boldsymbol{P}$ value \\
\hline Hypercholesterolemia & $8.13(1.22-54.37)$ & .03 \\
Length of stay (per day) & $1.03(1.01-1.05)$ & $<.01$ \\
\hline
\end{tabular}

14 , but also on an increased awareness and potentially lower clinical threshold to trigger a Doppler order (because DVTs were discovered on other days as well). In comparison, other modern single-institution studies on this topic reported an incidence rate ranging from $20 \%$ to $64 \%$, much higher than ours at $17 \%{ }^{4}$ In contrast, with other surgical procedures, such as heart transplantation and cardiac surgery, reported DVT incidences are lower at 5\%, and $14.8 \%$, respectively. ${ }^{1-3,8}$

In 2015, the International Society for Heart and Lung Transplantation reported an overall median survival of 5.7 years after LTx for primary transplantation. ${ }^{9}$ Significant pretransplant and peritransplant risk factors during the first posttransplant year that juxtapose with our study include age, male gender, type of underlying lung disease, time spent in the intensive care unit, and retransplantation; however, the occurrence of DVT was not among the outcomes studied (only the development of a pulmonary embolism was recorded). ${ }^{9,10}$ Although we found that the survival rates at the 1-year mark between patients who experienced DVT versus no DVT are not significantly different in the postoperative 90-day window, there exist significant survival differences between these groups after the 1-year mark and thus future studies are warranted to identify significant predictors of mortality in patients with and without DVTs. In an attempt to estimate the treatment effect of DVT screening, we conducted a propensity score match between the eras and found that patients who were screened trended toward having better 1-year survival. Combined with the increase in DVT detection, this suggests that long-term outcomes may offset the increased cost of screening, but future studies properly designed and powered are required to confirm this finding.

It is generally accepted that risk factors associated with the development of DVT during the postoperative period for any surgery include older age, medical illness, inherited thrombophilia, previous DVT, and longer immobilization 
Factors Associated with Deep Vein Thrombosis in Lung Transplant Surgery

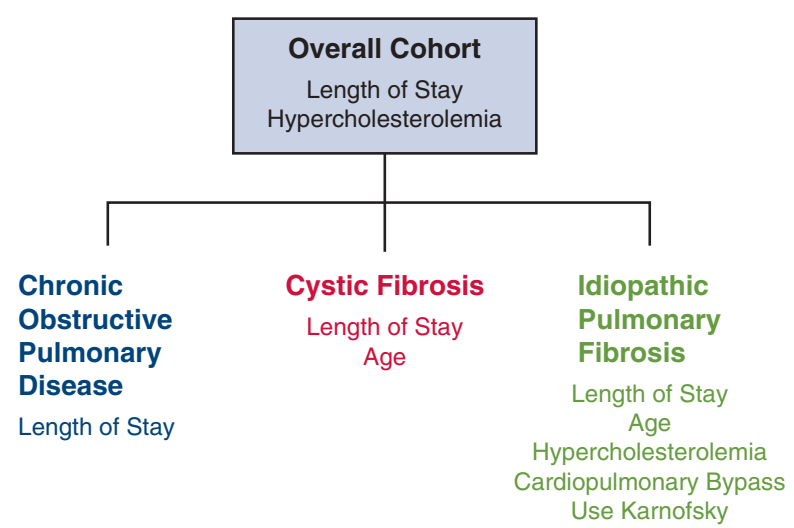

FIGURE 3. Visual summary of the risk factors associated with the development of DVT after lung transplantation. In the overall cohort, there was an association between an increased risk for the development of DVT with increased length of stay and in the presence of hypercholesterolemia. Length of stay remains associated with the 3 most-represented diagnoses, whereas other risk factors are differentially associated with each specific diagnosis as shown.

times. $^{4,8}$ In the LTx literature, 3 retrospective single institution reviews offer insight into this specific surgical population. Yegen and colleagues ${ }^{6}$ identified older age, postoperational pneumonia, and pretransplant DM diagnosis as predictors of a venous thromboembolism (121 total transplants with $20 \%$ DVT) in multivariable analysis, whereas Kahan and colleagues ${ }^{5}$ showed that only cardiopulmonary bypass was a notable predictor (153 total transplants with $29 \%$ DVT). Evans and colleagues ${ }^{4}$ identified bilateral LTx as significant in their univariable analysis (117 total transplants with $64 \%$ overall DVT). These results contrast with our findings in that pretransplant hypercholesterolemia and LOS were predictors of DVT. It is noteworthy that in univariable analyses, Kahan and colleagues ${ }^{5}$ showed that length of stay was a notable predictor of DVT.

In our univariable analysis, patients who were former tobacco smokers, when compared with patients who never smoked, were less likely to develop DVT in the overall group. In addition, we also observed that a diagnosis of COPD was associated with a lower probability of DVT. Diagnosis of idiopathic pulmonary fibrosis has been associated with an increase in DVT rates in other studies, ${ }^{11}$ and although we did not find a statistically significant difference, there was an increased incidence observed. Whereas almost all patients in the current study diagnosed with COPD were former smokers $(98 \% ; n=236)$, the majority of patients in the overall group were also former smokers $(69 \% ; n=500)$; thus, these 2 variables might not be necessarily surrogates of each other. On the other hand, a study recommending the routine use of DVT screening (after pneumonectomy) revealed a $9 \%$ incidence rate and that age and COPD were risk factors for the development of DVT. ${ }^{12}$ These observations are of interest and may be investigated in-depth during future work. Additionally, it is of interest that the subgroup of patients diagnosed with

TABLE 5. Characteristics of patients who developed second deep vein thrombosis (DVT)

\begin{tabular}{|c|c|c|c|c|c|c|c|c|c|}
\hline Diagnosis & $\begin{array}{l}\text { Time to } \\
\text { first } \\
\text { DVT (d) }\end{array}$ & Location* & $\begin{array}{l}\text { Time to } \\
\text { second } \\
\text { DVT (d) }\end{array}$ & Location & $\begin{array}{c}\text { Time to } \\
\text { death (mo) }\end{array}$ & $\begin{array}{c}\text { ECMO } \\
\text { use }\end{array}$ & $\begin{array}{c}\text { ICU } \\
\text { stay (d) }\end{array}$ & $\begin{array}{l}\text { Hospital } \\
\text { LOS (d) }\end{array}$ & PNA \\
\hline COPD & 2 & Right upper & 190 & Right lower & 21 & $\mathrm{~N}$ & 3 & 18 & $\mathrm{~N}$ \\
\hline COPD & 3 & Right upper & 233 & Right upper & 14 & $\mathrm{~N}$ & 18 & 24 & Y \\
\hline COPD & 31 & Right Lower & 201 & Right lower & - & $\mathrm{N}$ & 5 & 37 & $\mathrm{~N}$ \\
\hline $\mathrm{CF}$ & 4 & Left upper & 19 & Unknown & 3 & $\mathrm{~N}$ & 2 & 13 & $\mathrm{~N}$ \\
\hline $\mathrm{CF}$ & 24 & Right lower & 26 & Left upper & 5 & $\mathrm{~N}$ & 6 & 46 & $\mathrm{~N}$ \\
\hline $\mathrm{CF}$ & 81 & Left lower & 337 & Left lower & - & $\mathrm{N}$ & 3 & 19 & $\mathrm{~N}$ \\
\hline IPF & 1 & Right lower & 2 & Right upper & 54 & $\mathrm{Y}$ & 42 & 73 & $\mathrm{Y}$ \\
\hline IPF & 2 & Right lower & 33 & Right lower & - & $\mathrm{Y}$ & 17 & 40 & $\mathrm{~N}$ \\
\hline IPF & 3 & Right lower & 20 & Right upper & 6 & $\mathrm{Y}$ & 18 & 39 & $\mathrm{Y}$ \\
\hline IPF & 19 & Right upper & 124 & Left upper & 29 & $\mathrm{~N}$ & 5 & 35 & $\mathrm{Y}$ \\
\hline IPF & 20 & Left upper & 136 & Right upper & 13 & $\mathrm{~N}$ & 250 & 403 & $\mathrm{~N}$ \\
\hline IPF & 24 & Right upper & 594 & Unknown & 24 & $\mathrm{~N}$ & 30 & 55 & $\mathrm{Y}$ \\
\hline IPF & 24 & Left upper & 1337 & Unknown & 59 & $\mathrm{~N}$ & 59 & 201 & $\mathrm{Y}$ \\
\hline IPF & 36 & Right upper & 59 & Right lower & - & $\mathrm{Y}$ & 11 & 43 & $\mathrm{Y}$ \\
\hline Sarcoidosis & 22 & Bilateral lower & 401 & Left upper & 13 & $\mathrm{~N}$ & 96 & 183 & $\mathrm{~N}$ \\
\hline
\end{tabular}

$D V T$, Deep vein thrombosis; $E C M O$, extracorporeal mechanical oxygenation; $I C U$, intensive care unit; $L O S$, length of stay; $P N A$, pneumonia; $C O P D$, chronic obstructive pulmonary disease; $N$, no; $Y$, yes; $C F$, cystic fibrosis; $I P F$, idiopathic pulmonary fibrosis. *Location in the body (eg, right upper $=$ right upper body). 
idiopathic pulmonary fibrosis showed significant associations not only with LOS and hypercholesterolemia, but also with age, cardiopulmonary bypass use, and KPS score. Further studies should be carried out to identify this population to minimize DVT incidence with special attention to these significant variables (especially because LOS and hypercholesterolemia were significant in the multivariable analysis, as well). Of note, hypercholesterolemia has been correlated with the development of DVT with similar ORs and large $95 \%$ CI ranges. ${ }^{13,14}$ In our case, the percent of patients who had preoperative hypercholesterolemia and developed a DVT is disproportionately higher than those who did not. The total number of patients with preoperative high cholesterol levels is disproportionately low and thus the OR results in an increased risk $95 \% \mathrm{CI}$.

The subgroup composed of patients who developed a second DVT further support our discussion regarding variables studied earlier (Table 5). The mean postoperation intensive care unit stay for patients who developed a second DVT was 17 days, whereas in comparison the mean intensive care unit stay for the general cohort was 15 days. None of these patients had pretransplant hypercholesterolemia; however. Although the second DVT subgroup is too small to produce significant statistical studies, we were able to demonstrate that variables such as higher intensive care unit stays and higher LOS are remarkably associated with DVT outcomes.

The presence of DVT (asymptomatic or otherwise) early in a patient who has undergone LTx is associated with significant morbidity and mortality, as shown by our significantly different survival curves and median survival times. Additionally, there is a strong correlation between asymptomatic DVT occurrences and symptomatic venous thromboembolism episodes even with modern thromboprophylaxis ${ }^{15,16}$ and early complications have been associated with long-term morbidity and mortality. ${ }^{17}$ We found that universal DVT screening (in our case, at day 0 and day 14) significantly increased the rate of DVT detection and there was a trend toward screening resulting in improved 1 -year survival. Whereas this type of routine screening for asymptomatic patients or patients with nonspecific symptoms could be considered difficult and ineffective in general hospitalized populations, the use of these proven screening tools may be beneficial in post-LTx patient populations at a higher risk of DVT (eg, patients with a higher LOS or with other risk factors described above). The results of this study have influenced our clinical practice in that we now have lower thresholds to prompt our DVT protocols for patients displaying characteristics discussed in this investigation. Ultimately, we would need prospective clinical trials to assess the benefits of committing these patients to a specific anticoagulation protocol. These studies should include assessing the DVT-risk profile assessed in this investigation to not only indicate DVT protocol allocation, but also to reduce the likelihood or prevent a DVT before it occurs.
There are clear limitations to our study. Because ours was a retrospective cohort study, era effects and secular trends in medical and surgical care over the extensive study period by themselves can be expected to be producing improvements in outcomes over our study period. The nature of this study (eg, analyzing pre- and postprotocol changes) makes it impossible to adjust or control for this era effect and thus it is a significant limitation when interpreting the results seen in these groups. We have attempted to adjust for this using propensity matching across groups, but the analysis has the inherent limitation of including unmeasured confounders that may affect the treatment estimate. The collinear nature of this study is a significant limitation (eg, pre-DVT screenings were in a different year period than post-DVT screenings). In addition, the LOS and DVT association could reflect a sicker group potentially experiencing a higher complication rate. A future prospective study should have the power to distinguish between confounding variables of this nature. The long time span of this retrospective study could introduce further biases as well; for example, the common use of midline catheters and its confounding effects on upper DVT incidence. In addition, patients were only routinely screened for lower extremity DVTs and not upper extremity DVTs. We did not have any data on the false positive or false negative rates for detecting DVTs at our institution. Intraoperatively, all patients used compression stockings and sequential compression devices. These preventive measures were continued in the postoperative setting until the patient was discharged. Compliance with these was not actively measured. Upper extremity DVTs were only identified when there was clinical concern prompting evaluation. Moreover, important variables in the study of DVT pathogenesis such as inherited thrombophilias and acquired risk factors (eg, use of certain medications, renal disease, and previous DVT episode) were not studied directly. The increasing use of ECMO in our patients may have also contributed to some of the increase because when cannulas are removed, clot is a necessary and accepted consequence. To develop a more accurate model, a prospective study can be designed taking into consideration a fixed protocol for DVT surveillance with appropriate collection of these missing variables. Finally, all variables identified in this study are noted to be associated with the development of a DVT and given the limitations of the current retrospective study design, we are unable to make strong inferences about causality (eg, higher LOS increases DVT rates). We recognize that DVTs occurring following discharge are an important event in the postoperative course of patients undergoing LTx. Recently, the cancer literature has demonstrated that prophylactic enoxaparin is beneficial in routine practice for these high-risk patients. ${ }^{18}$ At this time, we have no specific discharge protocol recommendations based on our work for our patient population and the question of 
prophylaxis as it relates to LTx remains a subject for future investigations.

\section{CONCLUSIONS}

Universal DVT screening protocols have been shown to increase the rate of DVT detection in patients undergoing LTx. This increase in DVT screening may be associated with improved 1-year mortality. DVT continues to be an important contributor to morbidity and mortality in LTx populations. Important variables to consider identified in this study are LOS and pretransplant hypercholesterolemia, which were associated with DVT development. Recommendations to decrease a patient's risk for developing DVT such as striving to decrease their LOS are obvious; however, secondary preventative measurements against DVTs could be implemented in patients that carry a higher risk, such as universal screening protocols similar to those described in this study.

\section{Conflict of Interest Statement}

Authors have nothing to disclose with regard to commercial support.

The authors thank Ernest G. Chan, MD, MPH, for helping with the early development of this work.

\section{References}

1. Aziz F, Patel M, Ortenzi G, Reed AB. Incidence of postoperative deep venous thrombosis is higher among cardiac and vascular surgery patients as compared with general surgery patients. Ann Vasc Surg. 2015;29:661-9.

2. Alvarez-Alvarez RJ, Barge-Caballero E, Chavez-Leal SA, Paniagua-Martin MJ, Marzoa-Rivas R, Caamaño CB, et al. Venous thromboembolism in heart transplant recipients: incidence, recurrence and predisposing factors. J Heart Lung Transplant. 2015;34:167-74.

3. Ho KM, Bham E, Pavey W. Incidence of venous thromboembolism and benefits and risks of thromboprophylaxis after cardiac surgery: a systematic review and meta-analysis. J Am Heart Assoc. 2015;4:e002652.

4. Evans CF, Iacono AT, Sanchez PG, Goloubeva O, Kim J, Timofte I, et al. Venous thromboembolic complications of lung transplantation: a contemporary singleinstitution review. Ann Thorac Surg. 2015;100:2033-9.
5. Kahan ES, Petersen G, Gaughan JP, Criner GJ. High incidence of venous thromboembolic events in lung transplant recipients. J Heart Lung Transplant. 2007; 26:339-44.

6. Yegen HA, Lederer DJ, Barr RG, Wilt JS, Fang Y, Bagiella E, et al. Risk factors for venous thromboembolism after lung transplantation. Chest. 2007;132:547-53.

7. Schag CC, Heinrich RL, Ganz PA. Karnofsky performance status revisited: reliability, validity, and guidelines. J Clin Oncol. 1984;2:187-93.

8. Emuakhagbon V, Philips P, Agopian V, Kaldas FM, Jones CM. Incidence and risk factors for deep venous thrombosis and pulmonary embolus after liver transplantation. Am J Surg. 2016;211:768-71.

9. Yusen RD, Edwards LB, Kucheryavaya AY, Benden C, Dipchand AI, Goldfarb SB, et al. The registry of the International Society for Heart and Lung Transplantation: thirty-second official adult lung and heart-lung transplantation report-2015; focus theme: early graft failure. J Heart Lung Transplant. 2015;34:1264-77.

10. Lund LH, Edwards LB, Kucheryavaya AY, Dipchand AI, Benden C, Christie JD, et al. The registry of the International Society for Heart and Lung Transplantation: thirtieth official adult heart transplant report-2013; focus theme: age. $J$ Heart Lung Transplant. 2013;32:951-64.

11. Dalleywater W, Powell HA, Fogarty AW, Hubbard RB, Navaratnam V. Venous thromboembolism in people with idiopathic pulmonary fibrosis: a populationbased study. Eur Respir J. 2014;44:1714-5.

12. Raja S, Idrees JJ, Blackstone EH, He J, Badjatiya A, Mazzone P, et al. Routine venous thromboembolism screening after pneumonectomy: the more you look, the more you see. J Thorac Cardiovasc Surg. 2016;152: 524-32.e2.

13. Kawasaki T, Kambayashi J, Sakon M. Hyperlipidemia: a novel etiologic factor in deep vein thrombosis. Thromb Res. 1995;79:147-51.

14. Kawasaki T, Kambayashi J, Ariyoshi H, Sakon M, Suehisa E, Monden M. Hypercholesterolemia as a risk factor for deep-vein thrombosis. Thromb Res. 1997;88: $67-73$.

15. Ibrahim EH, Iregui M, Prentice D, Sherman G. Deep vein thrombosis during prolonged mechanical ventilation despite prophylaxis. Crit Care Med. 2002;30: $771-4$.

16. Ryu JH, Olson EJ, Pellikka PA. Clinical recognition of pulmonary embolism: problems of unrecognized and asymptomatic cases. Mayo Clin Proc. 1998;73.

17. Chan EG, Bianco V III, Richards T, Hayanga JW, Morrell M, Shigemura N, et al. The ripple effect of a complication in lung transplantation: evidence for increased long-term survival risk. J Thorac Cardiovasc Surg. 2016;151:1171-9.

18. Prins MH, Lensing AWA, Brighton TA, Lyons RM, Rehm J, Trajanovic M, et al. Oral rivaroxaban versus enoxaparin with vitamin $\mathrm{K}$ antagonist for the treatment of symptomatic venous thromboembolism in patients with cancer (EINSTEINDVT and EINSTEIN-PE): a pooled subgroup analysis of two randomised controlled trials. Lancet Haematol. 2014;1:e37-46.

Key Words: venous thromboembolism, lung transplantation, deep venous thrombosis, ultrasound screening, endstage lung disease 